\section{P033 IMPROVING PAEDIATRIC PRACTICE THROUGH MULTIDISCIPLINARY SAFETY HUDDLES}

Ka Yu Yung, Ruchi Sinha, Susan Giles. Imperial College Healthcare NHS Trust

10.1136/archdischild-2019-nppc.43

Background Medication is the most prevalent therapeutic intervention in patient management. ${ }^{1}$ Medication errors are incidents that have occurred in the medication cycle of prescribing, dispensing, administering, monitoring, or providing medicines advice, regardless of whether they caused harm. ${ }^{1}$ In a hospital setting, medication error rates are similar amongst adults and children but there is three times the potential to cause harm in the latter. Due to the complexities that are associated with prescribing for children and the potential for the lack of necessary metabolic reserves to buffer any consequences, ${ }^{2}$ ensuring high quality prescribing in paediatrics is paramount and this requires multidisciplinary team (MDT) collaboration. Pharmacy contributions: The introduction of weekly Safety Huddles was started on the Paediatric Intensive Care Unit (PICU) in March 2017 led by the PICU consultant, paediatric risk nurse and pharmacist. Safety Huddles are short MDT briefings, involving the ward-based medical, nursing \& pharmacy teams, providing a platform for all staff to understand things that are happening within the ward and anticipate further risks to improve patient safety and care. The aim is to create an open environment where staff regularly communicate and feel safe to raise concerns about patient safety. The Safety Huddle comprises of three main aspects: pharmacy updates as 'top tips'; Datix incident reports and issues/concerns of the week. Pharmacy interventions are collected on a daily basis and fed back to the individual prescriber immediately where possible as the exchange of information must be rapid to optimise engagement. These then form the weekly 'top tips' which are shared with the whole MDT, along with Datix reports and any particular concerns where learning and action points are developed and agreed through contribution by all.

Outcome The concept of Safety Huddles has been adapted and fully established throughout all paediatric and neonatal specialities at the Trust. There has been an increase in the number of incidents reported since the implementation of the Safety Huddles. Error themes and their impact are looked at so the team learn from improvement and harm occurrence or near misses. The measurement of interventions provides a weekly update to the individual team to see if these are being carried out effectively and to improve where necessary. It allows identification of triggers and incorporates problem solving through the involvement of all members of the team, improving staff, patient and family experience and communication in addition to reducing harm, allowing Trust values to be met. Lessons learned: Safety Huddles are held in the spirit of learning and improvement. It allows integration with the wider team, empowering the team to work unanimously towards the ultimate goal of delivering the best patient care.

\section{REFERENCES}

1. Department of Health and Social Care. The Report of the Short Life Working Group on reducing medication- related harm. February 2018

2. Department of Health. Medicines Standard: National Service Framework for Children, Young People and Maternity Services. October 2004.

3. Department of Health. Building a Safer NHS or Patients: Improving Medication Safety. January 2004.

\section{P034 \\ MANAGEMENT OF CHRONIC ILLNESS IN YOUNG PEOPLE AGED 10-24 YEARS: A SYSTEMATIC REVIEW TO EXPLORE THE ROLE OF PRIMARY CARE PHARMACISTS}

Mohammed Almunef, Julie Mason, Chris Curtis, Zahraa Jalal. School of Pharmacy, College of Medical and Dental Sciences, University of Birmingham

\subsection{6/archdischild-2019-nppc.44}

Aim To explore the role of primary care pharmacists in the management of chronic illnesses in young people aged 10-24 years.

Methods Systematic search of four databases: MEDLINE, EMBASE, Cochrane Library and CINAHL using MeSH (Medical Subject Heading) and Emtree terms covering three main themes, namely, pharmacist, young people and chronic illnesses. Inclusion criteria: articles identifying the role of primary care pharmacists in the management of chronic illness and its acute manifestations in young people aged 10-24 years. Exclusion criteria: articles referring to the hospital, secondary care setting. Chronic conditions such as disability. Acute disease. Conference abstracts, letters and case studies.

Results Eight relevant articles were identified from different countries UK(3), USA(3), Netherlands(1) and Chile(1). All of the articles made reference to community pharmacists only and did not include information on pharmacists working in any other area of primary care (e.g. general practice). Seven of the articles identified included original research studies (1 observational, 2 survey, 2 qualitative interview, 2 intervention). The remaining article was a literature review. The interventional studies utilised pharmacists to manage specific chronic illnesses (i.e. asthma and metabolic disease). Both showed significant results in terms of improvement of a young person's quality of life and improvement in their knowledge about their disease and its treatment. The research studies gathered the opinions of pharmacists (3) and young people (1) based on their experiences. The most prominent issue arising from the research into pharmacist opinion was the lack of direct communication with the young person because of parents collecting prescription items. In one study, ${ }^{1}$ around half of participants stated this to be the main cause of medication-related problems, which in the main were non-adherence, in young people. Community pharmacists identified many roles that they felt were of high priority to their practice when dealing with young people. These included supporting young people to develop generic health care skills, build trusting relationships directly with young people, counselling with affirmation on the necessity and benefits of adhering to their medicine regimens, helping young people to find credible online health information and use digital media for educational or reminder purposes, transferring information effectively across care interfaces and provision of specialist services. ${ }^{12}$

Conclusion There is a lack of published literature regarding the role of pharmacists in the management of chronic illness in young people. Where primary care pharmacists have managed chronic illnesses in this patient population, they have been community-based and have had a positive impact on patient outcomes. Pharmacists feel that they have a role to play in supporting young people with chronic illness and have identified many areas where they can provide additional services and support. However, many pharmacists perceive a fundamental communication barrier which hinders provision of this support i.e. lack of access to the patient. This suggests that given the appropriate training and access to these patients pharmacists could have a positive impact on patient outcomes. 